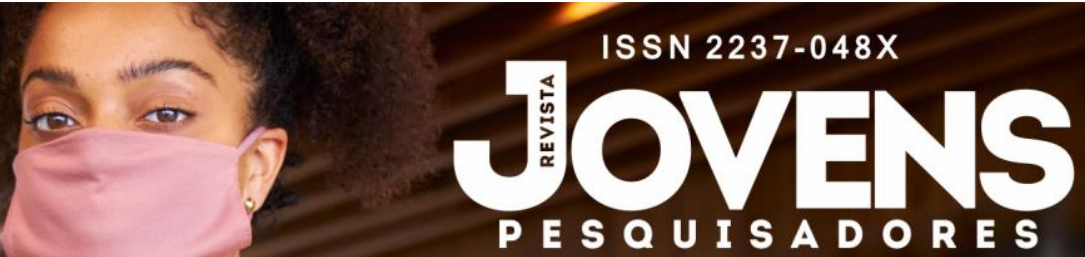

\title{
AS CONDIÇÕES DE SAÚDE DE IDOSOS RESIDENTES DO MEIO RURAL
}

\author{
ROSA, D. L. F'.; AREOSA, S.V.C. ${ }^{2}$
}

PALAVRAS-CHAVE: Envelhecimento humano. Velhice Rural. Saúde

\section{RESUMO}

Nas últimas décadas o Brasil se encontra em uma transição demográfica e epidemiológica, que passa pelo aumento da expectativa de vida da população, e consequentemente, do número de idosos. Neste sentido, 0 presente artigo objetivou verificar quais as condições de saúde de idosos residentes na zona rural do Município de Santa Cruz do Sul. Para isto, utilizou-se uma pesquisa transversal descritiva com abordagem quantitativa. Os dados apresentados foram obtidos a partir de um questionário socioeconômico e demográfico. Foram selecionados resultados acerca de problemas de saúde, doenças crônicas e a autopercepção da saúde. Ao todo participaram da pesquisa 236 pessoas idosas, entre 60 e 96 anos de idade. Acerca da autopercepção de saúde, $50,2 \%$ dos entrevistados afirmam possuir boas condições de saúde, demonstrando um bom índice de capacidade funcional, já que 51,2\% relatam não ter dificuldades na realização de tarefas da vida diária ou de trabalho. Apesar destes indicadores parecerem positivos, todos pesquisados alegam sofrer de algum tipo de doença crônicodegenerativa, como diabetes, hipertensão e problemas de coluna.

\section{HEALTH CONDITIONS OF ELDERLY PEOPLE LIVING IN RURAL AREAS}

\author{
KEYWORDS: Human Aging. Rural Old-Age. Health
}

\begin{abstract}
The demographic and epidemiological transition that reached Brazil brought with it an increase in life expectancy and, consequently, in the number of elderly people in the population. In this sense, this article aimed to verify the health conditions of elderly people living in the rural area of the Municipality of Santa Cruz do Sul. For this, crosssectional descriptive research with a quantitative approach was used, and the results presented were obtained from a socioeconomic and demographic questionnaire. Results about health problems, chronic diseases, and selfperceived health were selected. Altogether 236 elderly people, between 60 and 96 years old participated in the research. Regarding self-perceived health, $50.2 \%$ of respondents claim to have good health conditions, demonstrating a good index of functional capacity, since $51.2 \%$ report not having difficulties in performing tasks of daily living or work. Although these indicators seem positive, all surveyed claim to suffer from some type of chronic-degenerative diseases, such as diabetes, hypertension, and spine problems.
\end{abstract}

\footnotetext{
${ }^{1}$ Acadêmico do curso de Psicologia na Universidade de Santa Cruz do Sul. E-mail: di_fontoura@hotmail.com

2 Dra. em Serviço Social, Professora do Departamento de Psicologia, do Programa de Mestrado e Doutorado em Desenvolvimento Regional e Coordenadora do Mestrado Profissional em Psicologia na Universidade de Santa Cruz do Sul. E-mail: sareosa@unisc.br
} 


\section{INTRODUÇÃO}

O envelhecimento da população é um fenômeno global que está em alta escala, em curso principalmente nos países mais desenvolvidos. Isto é consequência de uma redução das taxas de fertilidade e mortalidade ocorridas ao longo das últimas décadas (FECHINE; TROMPIERI, 2012). 0 Brasil faz parte desta mudança demográfica, e já ao final da última década, a população brasileira com idade igual/ superior a 60 anos chegou a marca de 30 milhões segundo o Instituto Brasileiro de Geografia e Estatística (IBGE, 2017). Entretanto, o conhecimento das tendências no longo prazo das condições de saúde da população que atinge a velhice no país costuma se dirigir a mais informações relativas à mortalidade (LIMA-COSTA et al., 2011).

Em estudos primários sobre o aumento da população idosa, acreditava-se que as tendências da mortalidade representassem devidamente as condições de saúde dos idosos. Como a mortalidade estava em queda, assumia-se que as condições de saúde estavam melhores. Entretanto, estudos mais atuais demonstram que redução da mortalidade não implica necessariamente em anos adicionais com saúde e autonomia. É fundamental elucidar que a saúde da população idosa é multifatorial, e que neste sentido, a melhora de um aspecto não reflete necessariamente no outro (LIMA-COSTA et al., 2011). A autonomia da pessoa idosa diz respeito à sua saúde e à sua qualidade de vida, o que determina muito mais a forma como se envelhece do que a idade cronológica simplesmente. Isto quer dizer que ela está ligada a capacidade funcional destas pessoas, ou seja, na habilidade para desempenhar as atividades da vida diária (AVD). E prejuízos na capacidade funcional podem ser a maior razão para a perda da autonomia e da independência (RIGO; PASKULIN; MORAIS, 2010).

Alguns estudos têm privilegiado a lógica clínica no âmbito dos serviços de saúde e investigando danos e fragilidades específicas que acometem as pessoas idosas. Esses estudos trouxeram significativas contribuições, mas é necessário ir além para garantir qualidade de vida nessa etapa do ciclo vital (ROCHA et al., 2014). Assim, formou-se um consenso de que outros indicadores são importantes para se avaliar as condições de saúde na velhice. Entre esses indicadores, os mais comumente utilizados são a autoavaliação da saúde, a prevalência de doenças e de condições crônicas e a capacidade funcional (CAMPOLINA et al., 2013).

Com o intuito de contribuir para esta discussão o presente artigo apresentará um recorte do projeto de pesquisa intitulado "Estudo Socioeconômico e Demográfico da População Idosa no Meio Rural do Município de Santa Cruz do Sul", realizado pelo grupo de pesquisa "Envelhecimento e Cidadania" da Universidade de Santa Cruz do Sul através de financiamento captado junto ao Conselho Municipal do Idoso de Santa Cruz do Sul. 0 objetivo foi compreender as condições de saúde das pessoas idosas que vivem em zona rural, sendo este público menos favorecido tanto de recursos públicos, quanto de estudos relevantes, ocupando espaço geográfico em que se encontram realidades muito particulares, tanto econômicas, sociais quanto culturais (ALCÂNTARA, 2016).

Acredita-se que a relevância do presente estudo consiste no fato de que ainda são escassas as pesquisas sobre condições de vida e saúde das pessoas idosas em contextos rurais no Brasil. Assim, optou-se por estudar 
os indicadores de saúde (autoavaliação da saúde, prevalência de doenças crônico-degenerativas e capacidade funcional) e suas implicações na vida destes idosos.

\section{MATERIAIS E MÉTODOS}

Este é um estudo do tipo transversal descritivo de abordagem quantitativa, realizado nos anos de 2018 e 2019 em sete distritos rurais do município de Santa Cruz do Sul. Foi construído para a realização da coleta de dados um questionário misto, que passou por um estudo piloto em 2017 e foi elaborado a partir de um questionário socioeconômico e demográfico utilizado pelo Instituto Brasileiro de Geografia e Estatística (IBGE). Os dados selecionados para este artigo são relativos as respostas acerca das questões sobre saúde deste instrumento. No total participaram da pesquisa 236 idosos, 71 homens e 165 mulheres, entre 60 e 96 anos de idade. Essa amostra equivale a cerca de $10 \%$ do total de idosos em cada localidade, ou seja, dos distritos de Alto Paredão; Saraiva; São Martinho; Rio Pardinho; Boa Vista; Monte Alverne e São José da Reserva. Os questionários foram aplicados pelo grupo de pesquisa em Unidades Básicas de Saúde, Estratégias Saúde da Família (ESF), hospitais, grupos de convivência e recebeu ajuda de agentes comunitários nas localidades mais distantes para aplicar nas residências. Os resultados foram tratados através do software Statistical Package for Social Sciences (SPSS) - Versão 18.0 e são analisados com estatística descritiva, sendo apresentados em forma de tabelas.

\section{RESULTADOS E DISCUSSÃO}

Envelhecer, para a maioria no Brasil, é conviver com uma ou mais doenças crônicas (LIMA-COSTA et al., 2018). Para Tavares (2013) isto ocorre porque o processo está acelerado e as políticas públicas apresentam dificuldades para acompanhar. Por isso, é de suma importância o conhecimento da população sobre o papel da atividade física, alimentação balanceada e controle do peso, para que possíveis caminhos sejam traçados para a promoção da qualidade de vida e aumento das condições de saúde, além da redução de gastos públicos com o tratamento destas doenças (KNUTH et al., 2009). No final da década de 1990 o termo "envelhecimento ativo" foi adotado pela Organização Mundial da Saúde (OMS) relacionando o equilíbrio biopsicossocial e à capacidade que o idoso tem de desenvolver suas potencialidades. 0 envelhecimento ativo assume a abordagem de saudável, bem-sucedido, satisfatório, produtivo e, dependendo do contexto, apresentando-se como um novo paradigma acerca do envelhecimento e da velhice (BIDEL et al., 2016). Assim, boa parte da comunidade científica voltou-se para os temas, envelhecimento e saúde, enfocando aspectos preconizados pela OMS (2015) no que diz respeito à oferta de condições para desenvolvimento máximo das potencialidades das pessoas idosas (MARTINS et al., 2007).

Para conhecer a caracterização das condições de saúde dos idosos, faz-se mister obter informações detalhadas sobre diferentes aspectos da vida desses indivíduos que envelhecem. Uma forma de conduzir esse tipo de estudo é o levantamento de informações sobre a percepção das pessoas idosas em relação ao seu próprio estado de saúde (SILVA et al., 2012). Embora a medição do estado de saúde seja bastante difícil, por englobar diversos aspectos da vida do indivíduo, a autopercepção de saúde tem se mostrado um método confiável, e, 
recentemente, mais utilizado do que a observação direta para a análise desse aspecto (LINDEMANN et al., 2019). A autopercepção de saúde abrange aspectos da saúde física, cognitiva e emocional, se relacionando com a condição real ou objetiva de saúde (SILVA et al., 2012). A Tabela a seguir demonstra a autopercepção de saúde das pessoas idosas pesquisadas:

Tabela 1 - Autopercepção de saúde

\begin{tabular}{c|c}
\hline Considera que sua saúde é: & Percentual total \\
\hline Excelente & $2,1 \%$ \\
\hline Muito boa & $10,6 \%$ \\
\hline Boa & $39,6 \%$ \\
\hline Razoável & $40,4 \%$ \\
\hline Ruim & $6,4 \%$ \\
\hline Não respondeu & $0,9 \%$ \\
\hline Total & $100 \%$
\end{tabular}

Fonte: Estudo socioeconômico e demográfico da população idosa no meio rural do município de Santa Cruz do Sul, RS, 2019.

A maioria dos entrevistados avalia positivamente sua saúde $(52,3 \%)$ referindo sua saúde como boa $(39,6 \%)$, muito boa $(10,6 \%)$ ou excelente $(2,1 \%)$, também há um percentual grande de pessoas que avalia sua saúde como razoável $(40,4 \%)$ e apenas $6,4 \%$ a avalia como sendo ruim. Pode-se constatar que, de uma maneira geral, as pessoas idosas que participaram do estudo reconhecem a própria situação de saúde como adequada. Talvez, esse percentual de respondentes que afirmam que a saúde é boa, razoável estejam avaliando a mesma de forma positiva, pois podem estar levando em consideração às questões próprias do processo de envelhecimento, como as perdas que trazem dificuldades relacionadas ao bom funcionamento do corpo conforme a idade avança.

Em um estudo conduzido no interior do estado do Paraná (PILGER et al.,2011), a autopercepção da saúde atual foi considerada boa por $\mathbf{5 4 , 8 \%}$ da população idosa pesquisada, dados semelhantes com os encontrados no presente estudo. Já em uma revisão literária descrita por Gomes et al. (2019), alguns estudos demonstram que a presença de limitações em atividades de vida diária (AVDs), fatores socioeconômicos e presença de comorbidades, foram associados a percepções negativas de saúde. Este achado significa que a autonomia do idoso é um importante fator para a percepção da boa saúde e manutenção da qualidade de vida. Na Tabela seguinte se observa os dados sobre a autopercepção de saúde agora divididos por gênero:

Tabela 2 - Autopercepção de saúde por gênero

\begin{tabular}{c|c|c}
\hline Considera que sua saúde é: & Homens & Mulheres \\
\hline Excelente & $0,8 \%$ & $1,3 \%$ \\
\hline Muito boa & $3,4 \%$ & $7,2 \%$ \\
\hline Boa & $11 \%$ & $28,4 \%$ \\
\hline Razoável & $13,6 \%$ & $26,7 \%$ \\
\hline Ruim & $1,3 \%$ & $6,4 \%$ \\
\hline Não respondeu & $0 \%$ & $1,3 \%$
\end{tabular}


\begin{tabular}{c|c|c}
\hline Total & $30,1 \%$ & $69,9 \%$ \\
\cline { 2 - 3 } & Fonte: Estudo socioeconômico e demográfico da população idosa no meio rural do município de Santa Cruz do \\
Sul, RS, 2020.
\end{tabular}

Estes números acima apontam que no grupo feminino, a maior parte das idosas consideram sua saúde boa, muito boa ou excelente e uma minoria a considerou ruim $(5,1 \%)$. Em relação aos homens, também a maioria refere que sua saúde é boa, muito boa ou excelente, embora em percentuais bem inferiores ao do grupo feminino. No que diz respeito às diferenças de gênero é interessante ressaltar que as mulheres têm melhores cuidados com a saúde do que os homens. Diante disto, é possível perceber que há diferenças entre os gêneros e é essencial que as mesmas sejam compreendidas. Sabe-se, através de diversos estudos, que mesmo nos espaços urbanos os homens apresentam a tendência de buscar o serviço de saúde somente quando os problemas já estão agudos. No meio rural, esta situação se agrava; o acesso à saúde e a procura por estes espaços podem ser ainda menores (BURILLE; GERHARDT, 2014). Importante destacar que este fato se deve, não somente por empecilhos próprios do meio rural, mas também em razão daquilo que é culturalmente considerado masculino no campo, como a força e o trabalho. Assim, para muitos destes homens a ausência de saúde (ou a doença) podem significar o fim do trabalho, o que acarreta prejuízos diretamente no sentimento de pertencimento a um grupo, cujas características são historicamente definidas (BURILLE; GERHARDT, 2014). Os principais fatores determinantes da autopercepção de saúde contribuem para que sejam pensadas ações em prol da promoção da saúde e qualidade de vida de pessoas idosas.

Os estudos mais recentes ressaltam que a longevidade deve ser acompanhada de qualidade de vida e graus baixos de dependência. Assim, a condição que a pessoa idosa possui de viver de forma autônoma e de se relacionar em seu meio se refere a sua capacidade funcional. As perdas neste aspecto estão associadas ao maior risco de institucionalização e quedas, que podem comprometer a realização de tarefas diárias (RIGO; PASKULIN; MORAIS, 2010). 0 estudo realizado pelo ELSI (LIMA-COSTA et al., 2018) demonstra qual é o principal receio da população idosa brasileira: as quedas. De acordo com esta pesquisa, $43 \%$ dos idosos disseram ter medo de cair na rua e principalmente as consequências que deste fato, como possíveis dificuldades vindouras para realização de tarefas diárias. A capacidade funcional da pessoa idosa é influenciada por múltiplos fatores. Entre eles estão a existência de doenças crônicas, as características socioeconômicas e demográficas, a já citada autopercepção de saúde e a realização de atividades sociais (RIGO; PASKULIN; MORAIS, 2010). Avaliar a capacidade funcional é um grande desafio diante desta heterogeneidade, pois podem ocorrer defasagens físicas, cognitivas e/ou psíquicos capazes de privar as pessoas idosas a realizarem tarefas de autocuidado como higienizar-se e preparar alimentos sozinhos, anteriormente desempenhadas naturalmente (PIMENTA et al., 2014). Na tabela abaixo são demonstrados os resultados obtidos quando os idosos da amostra foram questionados acerca das dificuldades de realizar tarefas atuais:

Tabela 3 - Sente alguma dificuldade para realizar tarefas atuais?

\begin{tabular}{l|l} 
Não & 44,8 \\
\hline $\begin{array}{l}\text { Problemas nos membros } \\
\text { inferiores }\end{array}$ & 12,5
\end{tabular}




\begin{tabular}{l|l} 
Problemas na coluna & 12,1 \\
\hline $\begin{array}{l}\text { Problemas em outras partes do } \\
\text { corpo }\end{array}$ & 10,1 \\
\hline $\begin{array}{l}\text { Problemas nos membros } \\
\text { superiores }\end{array}$ & 3,2 \\
\hline Pressão alta & 2,8 \\
\hline Problema no sistema respiratório & 2,0 \\
\hline Problema no sistema nervoso & 2,0 \\
\hline $\begin{array}{l}\text { Problema no sistema esquelético e } \\
\text { muscular }\end{array}$ & 2,0 \\
\hline Depressão & 2,6 \\
\hline Diabetes & 1,2 \\
\hline Problemas de visão & 1,2 \\
\hline $\begin{array}{l}\text { Problemas do sistema } \\
\text { cardiovascular diferente da } \\
\text { hipertensão }\end{array}$ & 1,2 \\
\hline Outros problemas de saúde & 3,2 \\
\hline Total & 100,0
\end{tabular}

Fonte: Estudo socioeconômico e demográfico da população idosa no meio rural do município de Santa Cruz do Sul, RS, 2019.

Os números acima apontam que menos da metade das pessoas idosas entrevistadas (44,8\%) não apresentam dificuldade para realizar as tarefas da vida diária. Apesar destes indicadores apresentarem bons números, todos alegam sofrer com algum tipo de doença crônico-degenerativa, como diabetes, hipertensão e problemas de coluna. Problemas estes que afetam $70 \%$ da população idosa brasileira segundo o Estudo Longitudinal de Saúde dos Idosos Brasileiros- ELSI (LIMA-COSTA et al., 2018).

Os resultados de um estudo realizado no interior de Minas Gerais indicaram associação entre estas doenças em idosos e fatores como: não ter plano de saúde, morar sozinho (a), ter origem rural, pele não branca e baixa escolaridade (PIMENTA et al.,2014). Isso aponta para a necessidade de políticas públicas visando a promoção da saúde e prevenção de doenças, especialmente com os grupos mais vulneráveis. A fim de que, mesmo diante do envelhecimento populacional, indicadores de saúde possam melhorar. Os pesquisadores deste estudo ressaltam ainda dados relativos à diabetes, pois o avanço da idade aumenta o risco dessa doença. A diabetes pode diminuir significativamente a qualidade de vida do idoso, pois leva à morbidez e é uma das principais causas de mortalidade entre os idosos, podendo acarretar outras complicações, como insuficiência renal, amputação de membros, cegueira e doenças cardiovasculares (PIMENTA et al.,2014).

Outra questão que merece destaque é o tratamento oferecido para estas enfermidades, que geralmente são curso prolongado ou contínuo, acarretando gastos aos pacientes, suas famílias e sistema de saúde (Goulart, 2011). Além disso, alguns idosos não aceitam o diagnóstico e o tratamento, por não apresentarem sintomas ou por sentirem-se saudáveis (RIGO; PASKULIN; MORAIS, 2010). Tais situações favorecem a ocorrência da não adesão aos tratamentos. Neste sentido, o Ministério da Saúde colocou como prioridade na agenda do SUS a implementação de uma política para prevenção e promoção da saúde para as doenças crônicas mais prevalentes na velhice, com a elaboração do Plano de Ações Estratégicas, no Enfrentamento das Doenças Crônicas Não 
Transmissíveis no Brasil 2011-2022 (Brasil, 2011). Porém, com o grande número de idosos acometidos com doenças crônico-degenerativa pode-se considerar que os objetivos ainda não foram alcançados.

Vários fatores podem contribuir para acarretar tais diferenças de saúde e funcionalidade entre idosos: estilo de vida, aspectos socioeconômicos (incluindo educação, condição financeira, etnia e condições de trabalho) e o acesso a serviços de saúde. Outro ponto abordado pela pesquisa da ELSI (LIMA-COSTA et al., 2018) mostra que 75,3\% das pessoas idosas no país dependem do serviço do Sistema Único de Saúde (SUS), e que 83,1\% necessitaram ao menos uma consulta médica nos últimos 12 meses - incluindo a rede privada. Além da dificuldade de acesso aos serviços públicos, os idosos do meio rural em sua maioria não possuem condições financeiras de arcar com planos de saúde (TONEZER; TRZCINSKI; MAGRO, 2017 ), como se pode ver na amostra da pesquisa:

Tabela 4 - Planos de saúde

\begin{tabular}{c|c|c}
$\begin{array}{c}\text { Possui algum plano } \\
\text { de saúde? }\end{array}$ & Frequência & Porcentagem \\
\hline Sim & 89 & $37,7 \%$ \\
\hline Não & 146 & $61,9 \%$ \\
\hline Não respondeu & 1 & $0,4 \%$
\end{tabular}

Fonte: Estudo socioeconômico e demográfico da população idosa no meio rural do município de Santa Cruz do Sul, RS, 2019.

A maior parcela não possui qualquer plano de saúde, o que nos leva a considerar que essa porcentagem de pessoas idosas, $61,9 \%$, se encaixa com os demais estudos anteriormente citados, onde os serviços públicos do SUS são o ponto seguro de saúde desta população. Destaca-se aqui a importância das ESFs no contexto rural, isso porque, além de deslocar médicos e enfermeiros para locais mais distantes da sede do município, estas ainda auxiliam com orientações e informações à população, através de agentes comunitários de saúde. Ainda quanto ao acesso à saúde, sabe-se que este é fortemente influenciado pela escolaridade. Frequentemente os idosos que residem no ambiente rural são menos escolarizados, (a maioria dos idosos pesquisados na amostra têm apenas até quatro anos de estudo) e esta condição dificulta a identificação dos serviços de saúde. Este fator os deixa mais vulneráveis e possivelmente mais propensos aos riscos de adoecer. Atentar a estes detalhes é compreender que os aspectos, territoriais e culturais influenciam significativamente na saúde desta população.

Na tabela a seguir, são apresentados dados relativos ao acesso aos serviços de saúde, e observa-se que a grande maioria - 86\% - afirma ter em sua localidade uma Unidade Básica de Saúde (UBS) com Estratégia de Saúde da Família (ESF). No entanto, quanto às UBS Convencionais, o acesso diminui a menos da metade dos entrevistados $-48,7 \%$. No que diz respeito aos hospitais, quase a metade das pessoas idosas respondeu não ter acesso $-41,9 \%$.

Tabela 5 - Acesso à serviços de saúde na localidade rural

\begin{tabular}{l|l|l|l}
\hline Quais serviços de saúde você tem acesso? & Sim & Não & Não respondeu \\
\hline
\end{tabular}




\begin{tabular}{c|c|c|c}
$\begin{array}{c}\text { UBS com Estratégia de Saúde da Familia } \\
\text { (ESF) }\end{array}$ & $86 \%$ & $8,1 \%$ & $5,9 \%$ \\
\hline UBS Convencional & $48,7 \%$ & $24,6 \%$ & $26,7 \%$ \\
\hline Hospital & $32,6 \%$ & $41,9 \%$ & $25,4 \%$ \\
\hline
\end{tabular}

Fonte: Estudo socioeconômico e demográfico da população idosa no meio rural do município de Santa Cruz do Sul, RS, 2019.

Houve uma melhora do acesso da população rural brasileira aos serviços de saúde, e isto se deve ao crescimento da procura por estes serviços e da ampliação de postos e centros de saúde nestas regiões (TRAVASSOS; VIACAVA, 2007). Além disso, com os serviços oferecidos pelo SUS estas pessoas não precisam mais arcar com altas despesas no pagamento de serviços de saúde. Ainda assim, persistem desigualdades no acesso, principalmente em áreas rurais distantes e em localidades com poucos recursos e investimentos, que necessitam de um olhar mais acurado das autoridades de saúde do país (ROCHA et al., 2014).

\section{CONCLUSÃO}

Apesar de muitos estudos recentes serem direcionados a saúde da população idosa, observa-se que ainda existe uma carência quando se trata de políticas públicas voltadas as zonas rurais. Em nossa pesquisa e em outras realizadas em território nacional, constata-se que os atendimentos de saúde na rede pública são insuficientes. Ações que promovam um melhor acesso aos serviços de saúde e estratégias que favoreçam a inserção social, podem contribuir para a redução das taxas de prevalência e dependência funcional e para melhorar a saúde e a qualidade de vida destas pessoas idosas que vivem em contextos rurais. Sugerem-se outros estudos qualitativos que possam aprofundar o conhecimento sobre as diferenças de gênero em relação à percepção de saúde das pessoas idosas. Pudemos verificar que existem diferenças não apenas nos problemas apresentados, mas nas percepções sobre eles, mas um estudo qualitativo aprofundado poderia compreender de que forma ocorrem essas diferenças.

\section{AGRADECIMENTOS}

Agradecemos ao Conselho Nacional de Desenvolvimento Científico e Tecnológico (CNPQ) e a Fundação de Amparo à Pesquisa do Estado do Rio Grande do Sul (FAPERGS) as bolsas de iniciação científica para a participação de alunos de graduação. A Universidade de Santa Cruz do Sul (UNISC) e ao Fundo Municipal do Idoso de Santa Cruz do Sul pelos recursos financeiros que possibilitaram a realização da pesquisa. Os autores também agradecem aos profissionais da Estratégias Saúde da Família dos distritos e as pessoas idosas que se voluntariam a participar da pesquisa.

\section{REFERÊNCIAS}

ALCÂNTARA, A. Envelhecer no contexto rural: a vida depois do aposento. In: ALCÂNTARA, A.; CAMARANO, A.A; GIACOMIN, K.C. (Org). Políticas Nacional do Idoso: velhas e novas questões. Rio de Janeiro: IPEA, 2016. 
BIDEL R.M.R. et al. Envelhecimento ativo na concepção de um grupo de enfermeiros. Revista Kairós, São Paulo, v.19, n.22,p. 207-25,2016. Disponível em: <https://revistas.pucsp.br/index.php/kairos/article/view/32627>. Acesso em: 20 dez. 2019.

BRASIL. Secretaria de Vigilância em Saúde. Departamento de Análise de Situação de Saúde. Plano de ações estratégicas para o enfrentamento das doenças crônicas não transmissíveis (DCNT) no Brasil 2011-2022/ Ministério da Saúde. Brasília : Ministério da Saúde, 2011. Disponível em:

<http://bvsms.saude.gov.br/bvs/publicacoes/plano_acoes_enfrent_dcnt_2011.pdf>. Acesso em: 05 fev. 2020.

BURILLE, A.; GERHARDT, T. E. Doenças crônicas, problemas crônicos: encontros e desencontros com os serviços de saúde em itinerários terapêuticos de homens rurais. Saúde e Sociedade, São Paulo, v. 23, n. 2, p. 664-676, 2014. Disponível em: <http://www.revistas.usp.br/sausoc/article/view/84898/87634> . Acesso em 19 dez. 2019.

CAMARANO, A A.; FERNANDES, D. A previdência Social Brasileira. In: ALCÂNTARA, A. de O.; CAMARANO, A. A.; GIACOMIN, K. C. (Orgs.). Política Nacional do Idoso: velhas e novas questões. Rio de Janeiro: Ipea, 2016. CAMPOLINA, A.G. et al. A transição de saúde e as mudanças na expectativa de vida saudável da população idosa: possíveis impactos da prevenção de doenças crônicas. Caderno de Saúde Pública, Rio de Janeiro, v.29, n.6, p. 1217-1229, 2013.Disponível em: <https://www.scielo.br/scielo.php?pid=S0102311X2013000600018\&script=sci_abstract\&tIng=pt>. Acesso em: 17 jan. 2020.

FECHINE, B. R. A.; TROMPIERI, N.; 0 processo de envelhecimento: as principais alterações que acontecem com o idoso com o passar dos anos. Revista InterScience Place, Ceará, v. 1, n. 07, p. 106-132, 2012. Disponível em: <http://dx.doi.org/10.6020/1679-9844/2007>. Acesso em: 05 fev. 2020.

GOMES, G.C. et al. Fatores associados à autonomia pessoal em idosos: Revisão sistemática da literatura. Ciência e Saúde Coletiva, v.24, n.7, 2019. Disponível em:

< http://cienciaesaudecoletiva.com.br/artigos/fatores-associados-a-autonomia-pessoal-em-idosos-revisaosistematica-da-literatura/17269?id=17269> . Acesso em: 28 jan. 2020.

IBGE - Instituto Brasileiro de Geografia e Estatística. Pesquisa Nacional por Amostra de Domicílios Contínua. Rio de Janeiro: IBGE .2017. Disponível em:

<\&It;Https://agenciadenoticias.ibge.gov.br/media/com_mediaibge/arquivos/a7d023687b221aafb0364f56cad 94367.pdf \&gt>. Acesso em: 28 jan. 2020.

KNUTH A. G. et al. Conhecimento de adultos sobre o papel da atividade física na prevenção e tratamento de diabetes e hipertensão: estudo de base populacional no Sul do Brasil. Cadernos de Saúde Pública, Rio de Janeiro, v. 25, n. 3, p. 513-520, 2009. Disponível em:

<http://www.scielo.br/scielo.php?script=sci_arttext\&pid=S0102-311X2009000300006\&lng=en\&nrm=iso>. Acesso em: 03 fev. 2020.

LINDEMANN, I. L. et al . Autopercepção da saúde entre adultos e idosos usuários da Atenção Básica de Saúde. Ciência e Saúde Coletiva, Rio de Janeiro, v. 24, n. 1, p. 45-52, 2019. Disponível em: <http://www.scielo.br/scielo.php?script=sci_arttext\&pid=S1413-81232019000100045\&lng=en\&nrm=iso>. Acesso em: 03 fev. 2020.

LIMA-COSTA, M. F. et al. The Brazilian Longitudinal Study of Aging (ELSI-Brazil): Objectives and Design. American Journal of Epidemiology, Baltimore,V.187, n. 7, p.1345-1353, 2018. Disponível em:<

https://academic.oup.com/aje/article/187/7/1345/4831252> . Acesso em: 15 fev.2020.

et al . Tendências em dez anos das condições de saúde de idosos brasileiros: evidências da Pesquisa Nacional por Amostra de Domicílios (1998, 2003, 2008). Ciências e saúde coletiva, Rio de Janeiro, , v. 16, n. 9, p. 3689-3696, 2011. Disponível em: <http://www.scielo.br/scielo.php?script=sci_arttext\&pid=S141381232011001000006\&lng=en\&nrm=iso>. Acesso em: 13 dez. 2019. 
MARTINS, C.R. et al. Avaliação da qualidade de vida subjetiva dos idosos: uma comparação entre os residentes em cidades rurais e urbanas. Estudos Interdisciplinares em Envelhecimento, Porto Alegre, v.11, p.135-154, 2007. Disponível em: <https://seer.ufrgs.br/RevEnvelhecer/article/view/4817>. Acesso em: 12 dez. 2019.

MERA, C.M.P; NETTO, C.G.A. Envelhecimento dos produtores no meio rural na região do Alto Jacuí/RS e consequente migração para cidade. Estudos Interdisciplinares de Envelhecimento, Porto Alegre,v.19, n.3, p.75974, 2014. Disponível em: < https://seer.ufrgs.br/RevEnvelhecer/article/view/42871>. Acesso em: 17 jan. 2020.

MORAIS, E. P. de. et al. Os idosos mais velhos no meio rural: realidade de vida e saúde de uma população do interior gaúcho. Texto Contexto Enfermagem, Florianópolis, v. 13, n. 2, p. 374-383, 2008. Disponível em: <https://www.scielo.br/pdf/tce/v17n2/21.pdf>. Acesso em: 17 fev. 2020.

ORGANIZAÇÃo MUNDIAL DA SÁUDE. Relatório Mundial de Envelhecimento e saúde- Brasília: Organização PanAmericana da Saúde, 2015. Disponível em: <https://apps.who.int/iris/bitstream/handle/10665/186468/WHO_FWC_ALC_15.01_por.pdf?sequence=6>. Acesso em: 21 jan. 2020.

PILGER, C. et al. Características sociodemográficas e de saúde de idosos: contribuições para os serviços de saúde. Revista Latino-Americana de Enfermagem, Ribeirão Preto, v. 19, n. 5, p. 1230-1238, 201. Disponível em: <http://www.scielo.br/scielo.php?script=sci_arttext\&pid=S0104 11692011000500022\&lng=en\&nrm=iso>. Acesso em: 28 jan. 2020.

PIMENTA, F. B. et al . Fatores associados a doenças crônicas em idosos atendidos pela Estratégia de Saúde da Família. Ciência e Saúde Coletiva, Rio de Janeiro, v. 20, n. 8, p. 2489-2498, 2015. Disponível em: <http://www.scielo.br/scielo.php?script=sci_arttext\&pid=S1413-81232015000802489\&Ing=en\&nrm=iso>. Acesso em: 21 jan. 2020.

RIGO, I. I. et al. Capacidade funcional de idosos de uma comunidade rural do Rio Grande do Sul. Revista Gaúcha de Enfermagem, Porto Alegre, v. 31, n. 2, p. 254-261, 2010. Disponível em: <http://www.scielo.br/scielo.php?script=sci_arttext\&pid=S1983-14472010000200008\&lng=en\&nrm=iso>. Acesso em: 21 jan. 2020.

ROCHA, D,G. et al. Processo de revisão da Política Nacional de Promoção da Saúde: múltiplos movimentos simultâneos. Ciência e Saúde Coletiva, Rio de Janeiro, V.19,n.11,p. 4313-22,2014.Disponível em: <http://www.scielosp.org/pdf/csc/v19n11/1413-8123-csc-19-11-4313.pdf>. Acesso em: 12 dez. 2019. 
SILVA, R. J. dos S. et al . Prevalência e fatores associados à percepção negativa da saúde em pessoas idosas no Brasil. Revista Brasileira de epidemiologia, São Paulo, v. 15, n. 1, p. 49-62, 2012 . Disponível em <http://www.scielo.br/scielo.php?script=sci_arttext\&pid=S1415-790X2012000100005\&lng=pt\&nrm=iso>. Acesso em: 12 jan. 2020.

TAVARES, D.M.S. et al. Qualidade de vida e capacidade funcional de idosos residentes na zona rural. Revista de Enfermagem e Atenção à Saúde, Uberaba, v. 2, n. 1, 2013.

>http://seer.uftm.edu.br/revistaeletronica/index.php/enfer/article/viewFile/375/38 6>. Acesso em: 28 jan. 2020.

TONEZER, C.; TRZCINSKI, C. MAGRO, M. As Vulnerabilidades da Velhice Rural: Um Estudo de Casos Múltiplos no Rio Grande do Sul. Desenvolvimento em Questão, ljuí, v. 15, n. 40, p. 7-38. Disponível em: <https://www.revistas.unijui.edu.br/index.php/desenvolvimentoemquestao/article/view/5743> . Acesso em: 10 jan. 2020.

TRAVASSOS, C.; VIACAVA, F. Acesso e uso de serviços de saúde em idosos residentes em áreas rurais, Brasil, 1998 e 2003. Caderno Saúde Pública, Rio de Janeiro, v. 23, n. 10, p. 2490-2502, 2007 . Disponível em: <http://www.scielo.br/scielo.php?script=sci_arttext\&pid=S0102-311X2007001000023\&lng=en\&nrm=iso>. Acesso em: 09 fev. 2020. 